\title{
Local modalities for inoperable hepatocellular carcinoma: radiofrequency ablation versus stereotactic body radiotherapy
}

\author{
Sun Hyun Bae ${ }^{1}$, Hee Chul Park ${ }^{2}$ \\ ${ }^{1}$ Department of Radiation Oncology, Soonchunhyang University College of Medicine, Bucheon, Gyeonggi-do, Korea; ${ }^{2}$ Department of Radiation \\ Oncology, Samsung Medical Center, Sungkyunkwan University School of Medicine, Seoul, Korea \\ Correspondence to: Hee Chul Park, MD, PhD. Department of Radiation Oncology, Samsung Medical Center, Sungkyunkwan University School of \\ Medicine, Seoul, Korea. Email: hee.ro.park@skku.edu. \\ Provenance: This is a Guest Editorial commissioned by Section Editor Long Pan (Zhejiang Clinical Research Center of Minimally Invasive Diagnosis \\ and Treatment of Abdominal Diseases, Sir Run Run Shaw Hospital, Zhejiang University, Hangzhou, China). \\ Comment on: Rajyaguru DJ, Borgert AJ, Smith AL, et al. Radiofrequency Ablation Versus Stereotactic Body Radiotherapy for Localized \\ Hepatocellular Carcinoma in Nonsurgically Managed Patients: Analysis of the National Cancer Database. J Clin Oncol 2018;36:600-8.
}

Submitted Jul 25, 2018. Accepted for publication Aug 21, 2018.

doi: $10.21037 / \mathrm{atm} .2018 .08 .28$

View this article at: http://dx.doi.org/10.21037/atm.2018.08.28

Hepatocellular carcinoma (HCC) is one of the most common malignancies and a leading cause of cancer deaths in the worldwide (1). Liver transplantation is the only treatment option to cure both HCC and hepatic dysfunction simultaneously; however, because of limited availability of donor organs, transplantation is applied in a minority of patients who meet the Milan criteria (2). Hence, surgical resection is recommended as the first treatment option, although less than $30 \%$ of HCC patients are eligible at diagnosis owing to functional status, comorbidities, extent of disease, or hepatic decompensation in the setting of underlying liver disease (3). For unresectable HCC, patients are treated with various local ablative modalities, including radiofrequency ablation (RFA), stereotactic body radiotherapy (SBRT), microwave ablation, cryoablation, and percutaneous ethanol injection.

Among these, RFA is currently considered potentially curative and the best treatment option for early HCC (3-5). RFA achieves local control (LC) rates of 70-90\%. Several meta-analyses of randomized and nonrandomized controlled trials have shown that the effectiveness of RFA is comparable to that of surgical resection with fewer complications, especially in single HCC $\leq 3 \mathrm{~cm}(6,7)$. However, larger tumor size $(>3 \mathrm{~cm})$ and the existence of large abutting vessels $(\geq 3 \mathrm{~mm})$ lead to a reduction by approximately $50 \%$ in the rate of complete tumor necrosis (8). In addition, for tumor located close to the main biliary tree, abdominal organs, or the heart, the use of RFA is contraindicated because there is the possibility of severe complications, as well as the heat-sink effect (3). Therefore, an expert group from North America and Europe recommends that RFA be considered an established technique for the treatment of tumors that are limited in size $(\leq 3 \mathrm{~cm})$ and number $(\leq 3)$ and are located $\geq 1 \mathrm{~cm}$ away from critical organs and vessels (9).

Previously, radiotherapy (RT) has been used only in limited cases among HCC patients with palliative-intent aim because of low tolerance of the whole liver to RT. However, the introduction of three-dimensional conformal radiotherapy and further progress of RT techniques, including SBRT and intensity-modulated RT, have extended the indication of RT for the treatment of HCC to a curative-intent aim. SBRT is a "newly emerging treatment method to deliver a high dose of radiation to the target, utilizing either a single dose or a small number of fractions with a high degree of precision within the body" and several prospective SBRT studies have reported the efficacy of SBRT, with LC rates of $64-96 \%$ and overall survival (OS) rates of $34-72 \%$ at 2 years, which are comparable with other local modalities, as shown in Table 1 (10-15). Owing to a lack of level 1 evidence, however, SBRT is considered as an alternative treatment modality and not a standard treatment modality in most HCC guidelines $(3,5)$.

Recently, Rajyaguru et al. published a retrospective observational study comparing the effectiveness of RFA versus SBRT by using data of the National Cancer 
Table 1 Prospective studies of SBRT for hepatocellular carcinoma

\begin{tabular}{|c|c|c|c|c|c|c|c|c|c|}
\hline Authors & Study type & $\begin{array}{l}\text { No. of } \\
\text { pts }\end{array}$ & $\begin{array}{l}\text { CP class } \\
\text { A/B }(\%)\end{array}$ & $\begin{array}{l}\text { PVTT } \\
(\%)\end{array}$ & $\begin{array}{c}\text { Tumor size, } \\
\text { median } \\
\text { (range) }\end{array}$ & SBRT dose/fx & $\begin{array}{l}\text { F/u, months, } \\
\text { median (range) }\end{array}$ & LCR & OS \\
\hline $\begin{array}{l}\text { Kang et al. } \\
2012(11)\end{array}$ & Phase II & 47 & $87 / 13$ & 11 & $\begin{array}{c}2.9 \mathrm{~cm} \\
(1.3-7.8)\end{array}$ & $42-60 / 3$ & 17 [6-38] & $95 \%$ at 2 -year & $69 \%$ at 2-year \\
\hline $\begin{array}{l}\text { Lasley et al. } \\
2015 \text { (13) }\end{array}$ & Phase I/II & 59 & $64 / 36$ & 20 & $\begin{array}{c}33.6 \mathrm{~mL} \\
(2.0-107.3)\end{array}$ & $\begin{array}{c}\text { 36-48/3 for CPA; } \\
40 / 5 \text { for CPB }\end{array}$ & $\begin{array}{c}33.3(2.8-61.1) \\
\quad \text { for CPA; } \\
46.3(3.7-70.4) \\
\text { for CPB }\end{array}$ & $\begin{array}{c}91 \% \text { for CPA; } \\
82 \% \text { for CPB at } \\
\text { 3-year }\end{array}$ & $\begin{array}{l}72 \% \text { at } 2 \text {-year, } \\
61 \% \text { at } 3 \text {-year } \\
\text { for CPA; } 33 \% \text { at } \\
2 \text {-year, } 26 \% \text { at } \\
\text { 3-year for CPB }\end{array}$ \\
\hline $\begin{array}{l}\text { Takeda et al. } \\
2016(15)\end{array}$ & Phase II & 90 & $91 / 9$ & NS & $\leq 4 \mathrm{~cm}$ & $35-40 / 5$ & $41.7(6.8-96.2)$ & $96 \%$ at 3-year & $67 \%$ at 3-year \\
\hline
\end{tabular}

SBRT, stereotactic body radiotherapy; CP, Child-Turcotte-Pugh; PVTT, portal vein tumor thrombosis, LCR, local control rate; OS, overall survival; CPA, Child-Turcotte-Pugh A; CPB, Child-Turcotte-Pugh B; fx, fractions.

Table 2 Total doses and fractions of stereotactic body radiotherapy for stage I and stage II hepatocellular carcinoma

\begin{tabular}{lc}
\hline Parameters & No. of pts (\%) \\
\hline Total dose & $25[9]$ \\
$<30$ Gy & $35[13]$ \\
$30-39$ Gy & $115[42]$ \\
$40-49$ Gy & $60[22]$ \\
$\geq 50$ Gy & $40[14]$ \\
Unknown/missing & \\
Fractions & $26[9]$ \\
$1-2$ & $216[80]$ \\
$3-5$ & $17[6]$ \\
$\geq 6$ & $11[4]$ \\
Unknown/missing & $1[1]$ \\
Brachytherapy & \\
\hline
\end{tabular}

Database (NCDB), which includes about $70 \%$ of all newly diagnosed patients with cancer in the United States (16). Among 47,634 patients with HCC who had clinical stage I (T1N0M0) or stage II (T2N0M0) between 2004 and 2013, 3,684 (93\%) and 296 (7\%) nonsurgically managed patients received RFA and SBRT, respectively, as a primary treatment modality. Patients in the SBRT group were older ( $\geq 71$ years), Medicare recipients, had fewer comorbidity conditions according to Charlson-Deyo comorbidity score, and were more frequent in patients with stage II and tumor size $\geq 3 \mathrm{~cm}$, with statistical significance. Patients in the RFA group had more severe fibrosis score according to the Ishak fibrosis score, although underlying fibrosis data were unavailable for most patients (72\%). After propensity matching, the authors analyzed data of 521 patients in the RFA group and 275 patients in the SBRT group. SBRT doses and fractions are presented in Table 2. The followup period for all patients ranged from 14.1 to 41 months 
(median, 25.3 months). The 5-year OS was 30\% [95\% confidence interval (CI), $24.5 \%$ to $35.3 \%$ ] in the RFA group and $19 \%$ (95\% CI, $13.5 \%$ to $25.9 \%$ ) in the SBRT group [hazard ratio (HR), $0.67 ; 95 \% \mathrm{CI}, 0.55$ to $0.81 ; \mathrm{P}<0.001$ ]. They conducted sensitivity analysis to evaluate the potential effects by unmeasured severe fibrosis and found consistent results; the advantage of RFA over SBRT was relatively robust. The authors suggested that treatment with RFA yields superior survival compared with SBRT for stage I or stage II HCC.

On the other hand, Wahl et al. showed that both RFA and SBRT are effective local modalities for inoperable HCC, using the retrospective data from the University of Michigan (17). Between 2004 and 2012, among a total 224 patients with inoperable and nonmetastatic HCC, 161 patients received RFA for 249 tumors and 63 patients received SBRT for 83 tumors. The SBRT group had lower rates of liver cirrhosis, lower Child-Pugh (CP) scores, higher rates of alpha-fetoprotein, and more previous liverdirected therapies, with statistical significance. SBRT doses were $27-60 \mathrm{~Gy}$, in 3 (46\%) or 5 (53\%) fractions; the median biologically effective dose (BED) for all patients was $100 \mathrm{~Gy}_{10}$, assuming an $\alpha / \beta$ ratio of 10 . The median followup times were 20 months in the RFA group and 13 months in the SBRT group. The 1 and 2 -year LC rates were $84 \%$ and $80 \%$ in the RFA group and $97 \%$ and $84 \%$ in the SBRT group, respectively. The 1 and 2-year OS rates were $70 \%$ and $53 \%$ in the RFA group and $74 \%$ and $46 \%$ in the SBRT group, respectively, with no statistical significance. In inverse probability of treatment weighting univariate analysis, increased tumor size predicted local recurrence with RFA (HR, 1.54 per cm; $\mathrm{P}=0.006$ ) but not with SBRT (HR, 1.21 per $\mathrm{cm} ; \mathrm{P}=0.617$ ). For tumors $<2 \mathrm{~cm}$, there was no significant difference between RFA and SBRT in LC rate (HR, 2.50; 95\% CI, 0.72 to $8.67 ; \mathrm{P}=0.15$ ); however, for tumors $\geq 2 \mathrm{~cm}, \mathrm{LC}$ rate was significantly decreased in the RFA group (HR, 3.35; 95\% CI, 1.17 to 9.62; $\mathrm{P}=0.025$ ). The authors suggested that both modalities provide excellent LC for small HCC but that SBRT may have an advantage for tumors $\geq 2 \mathrm{~cm}$. Although there are no randomized trial data comparing RFA and SBRT, the authors' conclusion coincides with historical studies.

Therefore, the conclusive and dissimilar remarks from the abovementioned observational study using NCDB has prompted several debates (18). First, there are intrinsic limitations of the NCDB. The data did not include Eastern Cooperative Oncology Group status, underlying liver function (CP score), Barcelona Clinic Liver Cancer stage, tumor size, tumor thrombosis or vascular invasion, tumor location (central, subphrenic, or perivascular area), inability to tolerate invasive procedures (pacemakers or defibrillators, contraindication to sedation), and proper subsequent treatments. These unmeasured confounders significantly affect OS in HCC patients. Practically, the main reasons for SBRT referral are poor performance status, larger tumor size, portal vein tumor thrombosis (PVTT), and tumors near large vessels; the unmeasured imbalances between RFA and SBRT could not be corrected because a propensitymatching technique did not account for unobserved factors in the NCDB study. In addition, SBRT was applied in only $7 \%$ of patients of the NCDB data; the small sample size in the SBRT group may not be representative. Lastly, collected data from registered facilities has not been systematically reviewed or validated, and registry-to-registry variations in the reliability and accuracy of NCDB data are possible. Second, both RFA and SBRT are local modalities. The failure patterns of HCC are not only local recurrences but also intrahepatic recurrences. Application of subsequent treatments for multiple intrahepatic tumors after initial treatment is important, to prolong survival. Therefore, small differences in LC between RFA and SBRT would have little effect on OS. LC seems to be more representative than OS as the primary end point to determine the efficacy of a local modality. Third, SBRT was conducted from 2004 to 2014. The period prior to 2010 was in the early phase of development and optimization of SBRT. Overall quality assurance of SBRT planning and delivery was not described in the study. One patient treated with brachytherapy was included. The SBRT dose was unknown in $14 \%$ of patients and $\leq 39$ Gy in $22 \%$ : $36 \%$ of patients in the SBRT group did not receive standard doses. The SBRT fraction was 1-2 in $9 \%$ of patients. Lower total dose and $\leq 2$ fractions would suggest that SBRT was conducted with a palliative aim. Inappropriate SBRT technique could certainly contribute to decreased OS.

To strengthen the evidence of SBRT for HCC, there are several considerations. First, there is no clear evidence for the minimal effective dose or the maximal tolerable dose of SBRT in HCC. Table 1 shows various fractionation schemes among prospective SBRT studies, although these studies reported the promising treatment outcomes. Jang et al. suggested a dose-response relationship for LC after SBRT using 3 fractions in HCC (19). To achieve 2-year LC with a probability of $90 \%$, authors assumed that a required SBRT dose was 51.1 Gy for tumor size $\leq 5 \mathrm{~cm}$ (95\% CI, 47.7 to $54.5)$ and 61.2 Gy for tumor size $>5 \mathrm{~cm}(95 \% \mathrm{CI}, 58.4$ to 
64.0), respectively. On the other hand, Wahl et al. suggested that the use of sufficiently ablative RT doses (median $\left.\mathrm{BED}=100 \mathrm{~Gy}_{10}\right)$ showed no dose-response relationship for LC in cases of $\mathrm{HCC} \leq 5 \mathrm{~cm}$ (17). A recent systematic quantitative review of 13 liver SBRT studies reported that the LC rates for primary liver tumors were $93 \%$ at 1 year, $89 \%$ at 2 -year, and $86 \%$ at 3 -year, respectively. There was no dose-response relationship for LC among BED $60-180 \mathrm{~Gy}_{10}$ (20). Further studies based on larger populations are needed to specify the optimal SBRT doses: the minimal effective dose for small tumors $\leq 5 \mathrm{~cm}$ and the maximal tolerable dose for large tumors $>5 \mathrm{~cm}$. Second, SBRT studies treated HCC patients with various inclusion criteria, because patients who were unsuitable for RFA or other local modalities mainly referred for SBRT as an alternative treatment option. Unfavorable factors including tumor $>3 \mathrm{~cm}$ and PVTT significantly affect intrahepatic recurrence and OS, resulting in inconsistent treatment outcomes among studies. Nevertheless, some cost-effectiveness studies have showed similar outcomes between RFA and SBRT $(21,22)$. In future, the target population should be subdivided, to determine optimal indications. SBRT study of small HCC without vascular invasion would focus on comparable efficacy with RFA. However, SBRT study of larger-sized HCC or vascular invasion, which include potential risk of recurrence or metastases, might improve treatment outcome using combined modalities such as transarterial chemoembolization, sorafenib, or immune therapy. Third, prospective multicenter studies using SBRT in HCC have not yet been published. Single-center studies have smaller and more uniform sample sizes and unique practice patterns. On the other hand, multicenter study includes a more heterogeneous sample of participants and a certain degree of diverse practice in the real world. This provides better generalizability and representativeness of the study results, and is therefore essential for SBRT to achieve standardization. It is expected that the results of several ongoing prospective multicenter studies, registered on www.clinicaltrials.gov (NCT01850667, NCT0 1850368 , NCT03172559, NCT02470533, NCT02958163, and NCT01730937), will provide answers to some of these issues.

\section{Conclusions}

Many prospective and retrospective studies have reported that SBRT shows a comparable efficacy with that of RFA. However, an NCDB observational study concluded that RFA yields superior survival compared with SBRT for stage
I or stage II HCC. This result has given rise to several debates because of intrinsic limitations of unmeasured confounders, highlighting the need for prospective randomized trials in the field of radiation oncology. No single treatment modality can be recommended to all patients with HCC, necessitating a multidisciplinary approach to make a tailored treatment plan on the basis of the performance status, comorbidities, severity of hepatic decompensation, tumor extent, presence of metastasis, cancer-related symptoms, and patient preference (23). Therefore, further prospective, multicenter studies will be needed to determine the optimal indications, to maximize the efficacy of SBRT and standardize SBRT techniques, which will result in a treatment option that is equivalent to other local modalities in HCC treatment guidelines.

\section{Acknowledgements}

Funding: This research was supported by the Marine Biotechnology Program funded by Ministry of Oceans and Fisheries, Korea (20150220) and by the Soonchunhyang University Research Fund.

\section{Footnote}

Conflicts of Interest: The authors have no conflicts of interest to declare.

\section{References}

1. Jemal A, Bray F, Center MM, et al. Global cancer statistics. CA Cancer J Clin 2011;61:69-90.

2. Mazzaferro V, Regalia E, Doci R, et al. Liver transplantation for the treatment of small hepatocellular carcinomas in patients with cirrhosis. N Engl J Med 1996;334:693-9.

3. Marrero JA, Kulik LM, Sirlin CB, et al. Diagnosis, Staging and Management of Hepatocellular Carcinoma: 2018 Practice Guidance by the American Association for the Study of Liver Diseases. Hepatology 2018;68:723-50.

4. Korean Liver Cancer Study G, National Cancer Center K. 2014 KLCSG-NCC Korea Practice Guideline for the Management of Hepatocellular Carcinoma. Gut Liver 2015;9:267-317.

5. European Association for the Study of the Liver. Electronic address eee, European Association for the Study of the L. EASL Clinical Practice Guidelines: Management of hepatocellular carcinoma. J Hepatol 2018;69:182-236. 
6. Wang Y, Luo Q, Li Y, et al. Radiofrequency ablation versus hepatic resection for small hepatocellular carcinomas: a meta-analysis of randomized and nonrandomized controlled trials. PLoS One 2014;9:e84484.

7. Jia JB, Zhang D, Ludwig JM, et al. Radiofrequency ablation versus resection for hepatocellular carcinoma in patients with Child-Pugh A liver cirrhosis: a meta-analysis. Clin Radiol 2017;72:1066-75.

8. Lencioni R, Crocetti L. Local-regional treatment of hepatocellular carcinoma. Radiology 2012;262:43-58.

9. Lencioni R, de Baere T, Martin RC, et al. Image-Guided Ablation of Malignant Liver Tumors: Recommendations for Clinical Validation of Novel Thermal and NonThermal Technologies - A Western Perspective. Liver Cancer 2015;4:208-14.

10. Mendez Romero A, Wunderink W, Hussain SM, et al. Stereotactic body radiation therapy for primary and metastatic liver tumors: A single institution phase i-ii study. Acta Oncol 2006;45:831-7.

11. Kang JK, Kim MS, Cho CK, et al. Stereotactic body radiation therapy for inoperable hepatocellular carcinoma as a local salvage treatment after incomplete transarterial chemoembolization. Cancer 2012;118:5424-31.

12. Bujold A, Massey CA, Kim JJ, et al. Sequential phase I and II trials of stereotactic body radiotherapy for locally advanced hepatocellular carcinoma. J Clin Oncol 2013;31:1631-9.

13. Lasley FD, Mannina EM, Johnson CS, et al. Treatment variables related to liver toxicity in patients with hepatocellular carcinoma, Child-Pugh class A and B enrolled in a phase 1-2 trial of stereotactic body radiation therapy. Pract Radiat Oncol 2015;5:e443-9.

14. Scorsetti M, Comito T, Cozzi L, et al. The challenge of inoperable hepatocellular carcinoma (HCC): results of a single-institutional experience on stereotactic body radiation therapy (SBRT). J Cancer Res Clin Oncol 2015;141:1301-9.

15. Takeda A, Sanuki N, Tsurugai Y, et al. Phase 2 study of

Cite this article as: Bae SH, Park HC. Local modalities for inoperable hepatocellular carcinoma: radiofrequency ablation versus stereotactic body radiotherapy. Ann Transl Med 2018;6(Suppl 1):S3. doi: 10.21037/atm.2018.08.28 stereotactic body radiotherapy and optional transarterial chemoembolization for solitary hepatocellular carcinoma not amenable to resection and radiofrequency ablation. Cancer 2016;122:2041-9.

16. Rajyaguru DJ, Borgert AJ, Smith AL, et al. Radiofrequency Ablation Versus Stereotactic Body Radiotherapy for Localized Hepatocellular Carcinoma in Nonsurgically Managed Patients: Analysis of the National Cancer Database. J Clin Oncol 2018;36:600-8.

17. Wahl DR, Stenmark MH, Tao Y, et al. Outcomes After Stereotactic Body Radiotherapy or Radiofrequency Ablation for Hepatocellular Carcinoma. J Clin Oncol 2016;34:452-9.

18. Rajyaguru DJ, Borgert AJ, Halfdanarson TR, et al. Reply to E.L. Pollom et al, N. Ohri et al, A. Fiorentino et al, D.R. Wahl et al, N. Kim et al, J. Boda-Heggemann et al, S. Rana et al, N. Sanuki et al, J.R. Olsen et al, G.L. Smith et al, and A. Shinde et al. J Clin Oncol 2018;36:2567-9.

19. Jang WI, Kim MS, Bae SH, et al. High-dose stereotactic body radiotherapy correlates increased local control and overall survival in patients with inoperable hepatocellular carcinoma. Radiat Oncol 2013;8:250.

20. Ohri N, Tome WA, Mendez Romero A, et al. Local Control After Stereotactic Body Radiation Therapy for Liver Tumors. Int J Radiat Oncol Biol Phys 2018. [Epub ahead of print].

21. Seo YS, Kim MS, Yoo HJ, et al. Radiofrequency ablation versus stereotactic body radiotherapy for small hepatocellular carcinoma: a Markov model-based analysis. Cancer Med 2016;5:3094-101.

22. Pollom EL, Lee K, Durkee BY, et al. Cost-effectiveness of Stereotactic Body Radiation Therapy versus Radiofrequency Ablation for Hepatocellular Carcinoma: A Markov Modeling Study. Radiology 2017;283:460-8.

23. Siddique O, Yoo ER, Perumpail RB, et al. The importance of a multidisciplinary approach to hepatocellular carcinoma. J Multidiscip Healthc 2017;10:95-100. 